\title{
Using Exponential Finite Difference Method for Solve Kuramoto-Sivashinsky Equation with Numerical Stability Analysis
}

Abdulghafor M. Al-Rozbayani

Shrooq M. Azzo

Department of Mathematics,

College of Computer Sciences and Mathematics, University of Mosul, Iraq

\section{Received on: 30/01/2013}

\section{ABSTRACT}

Accepted on: 03/04/2013

In this paper we solved the Kuramoto-Sivashinsky Equation numerically by finitedifference methods, using two different schemes which are the Fully Implicit scheme and Exponential finite difference scheme, because of the existence of the fourth derivative in the equation we suggested a treatment for the numerical solution of the two previous scheme by parting the mesh grid into five regions, the first region represents the first boundary condition, the second at the grid point $\mathrm{x} 1$, while the third represents the grid points $\mathrm{x}_{2}, \mathrm{x}_{3}, \ldots \mathrm{x}_{\mathrm{n}-2}$, the fourth represents the grid point $\mathrm{x}_{\mathrm{n}-1}$ and the fifth is the second boundary condition. We also, studied the numerical stability by Fourier (Von-Neumann) method for the two scheme which used in the solution on all mesh points to ensure the stability of the point which had been treated in the suggested style, we using two interval with two initial condition and the numerical results obtained by using these schemes are compare with Exact Solution of Equation Excellent approximate is found between the Exact Solution and numerical Solutions of these methods.

Keywords: Kuramoto-Sivashinsky Equation, Fully Implicit scheme, Exponential finite difference scheme, Von-Neumann method.

استخدام طريقة الفروقات المنتهية الأسية لحل معادلة Kuramoto-Sivashinsky مع تحليل الاستقرارية

العددية

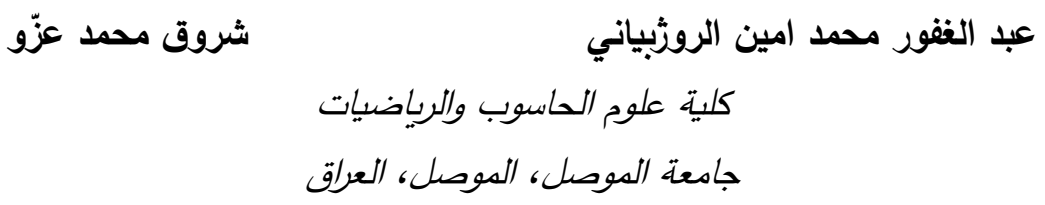

في هذا البحث تم حل معادلة Kuramuto-Sivashinsky بطرائق الفروقات المنتهية عددياً, وقد استخدمت طريقتان وهي الطريقة الضمنية الكاملة وطريقة الفروقات المنتهية الأسية, ولكون المعادلة تحتوي على المشتقة الرابعة فقد اقترحت معالجة للحل العددي للطريقتين, بتجزئة نقاط المشبك إلى خمس مناطق الأولى الثرط الحدودي

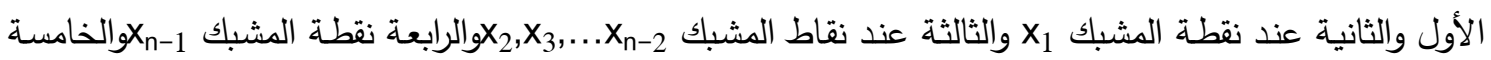

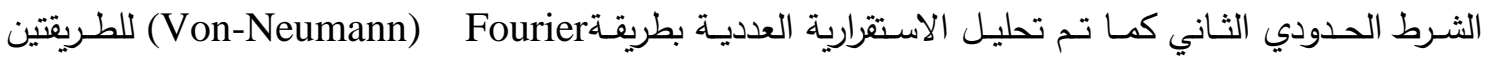
المستخدمة في الحل وعلى نقاط المشبك كافة للتأكد من استقرارية النقاط التي عولجت بالأسلوب المقترح, وقد أخذت

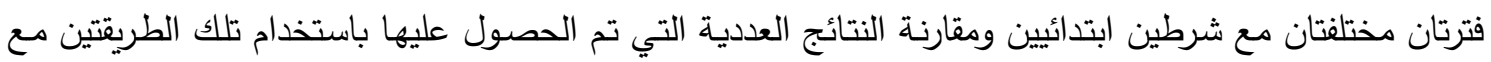
الحل المضبوط للمعادلة وقد وجدت تقريباً ممتازاً بين الحل المضبوط والحلول العددية لتلك الطرائق.

الكلمـات المفتاحيـة: معادلة Kuramuto-Sivashinsky, الطريقـة الضمنية الكاملة , طريقـة الفروقات المنتهيـة الأسية, طريقة فون نيومان. 


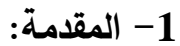

أصبحت المعادلات التفاضلية الجزئية أداة مفيدة لوصف الظواهر الطبيعية لنماذج العلوم والهندسة, لذلك أصبح من الضروري حل هذه المعادلات التفاضلية الجزئية باستخدام الطرائق العددية والمتطورة, وتطبيق هذه الطرائق[15].

وتظهر المعادلات التفاضلية الجزئية في بعض فروع الرياضيات التطبيقية, وتعد الديناميكية السائلة للماء و ميكانيكية الكم و النظرية الكهرومغناطيسية أمثلة للمعادلات التفاضلية الجزئية, وتعد المعالجة التحليلية لهذهاه المعادلات عملية معقدة جداً إذ تتطلب تطبيق الطرائق الرياضية المتقدمة, من ناحية أخرى أصبح استخدام الطرائق العددية البسيطة والكفوءة عموماً أسهل لإيجاد حلول تقريبية بما فيه الكفاية[12]. درس العديد من الباحثين معادلة Kuramoto-Sivashinskyببب تعقيدها وقدرتها على وصف تشكيلة كبيرة من الظواهر الطبيعية مثل استقرار جبهات الأغشية المنحدرة لذلك هذه المعادلة جذبت الكثير من الانتباه في المجاميع العلمية [3].

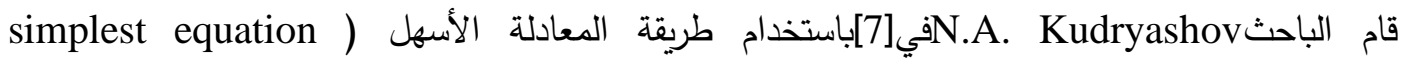
(method) للبحث عن حلول مضبوطة (exact solution) من طريقة المعادلات التفاضلية غير الخطية الجديدة, وتقدم للبحث عن حلول مضبوطة من المعادلات التقاضلية غير الخطية, وهناك فكرتان أساسيتان في البحث

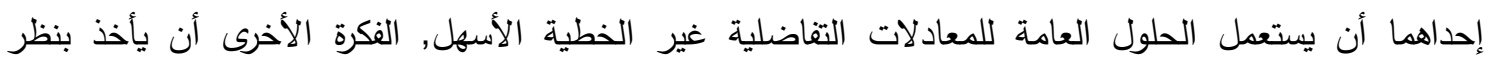
الاعتبار المعادلات المفردة المحتملة للدرس, وتستعمل الطريقة لإيجاد حلول مضبوطة لمعادلة K.S والمعادلة توصف الموجات غير الخطية في السائل الموصل للحرارة.

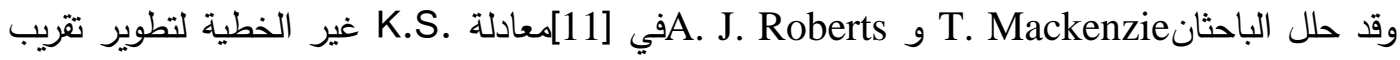
الفروقات المنتهية إلى حركية, وكان التحليل مستنداً على النظرية المنوعة المركزية (center manifold theory) وقد كانوا مطينّين لأن أنموذج الفروقات المنتهية تنظم الحركة بدقة وقد تُرتب بشكل منظم, إن النظرية تُطبق بعد K.S. تقسيم المجال الطبيعي إلى عناصر صغيرة بإدخال عناصر الحدود الداخلية التي تحذف لاحقاً, إن معادلة استعملت مثالاً لإثبات أن الفروقات المنتهية الثمولية قد تُطبق على معادلات الرتبة الرابعة غير الخطية, وقد أثبتوا أن النظرية المنوعة المركزية المبتكرة شمولية بمعنى أنها تعالج المعادلات الحركية ككل ليس فقط كمجموع الثروط لئه

أما الباحثانAAl-Baker. و Al-Rawi] فقد قاما بحل معادلة Korteweg-de Vries-Burger's باستخدام طريقتين من طرائق الفروقات المنتهية وهما الطريقة الضمنية الكاملة وطريقة الفروقات المنتهية الأسية, ودرسوا الاستقرارية العددية بطريقة Fourier(Von-Neumann) للطريقتين وعلى كافة نقاط المشبك, وقارنوا النتائج العددية التي تم الحصول عليها باستخدام تلك الطرائق مع نتائج الحل المضبوط, ووجدوا تقارباً بين الحل التحليلي والحلول العددية للطريقتين.

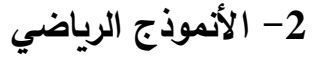

إن صيغة معادلة Kuramoto-Sivashinsky تكون على النحو الآتي [10] :

$u_{t}=-u u_{x}-u_{x x}-u_{x x x x} \quad,[0,32 \pi]$

$$
\text { والشروط الحدودية هي }
$$




$$
\begin{aligned}
& \left.\begin{array}{ll}
u(0, t)=u(32 \pi, t)=1 & , t \geq 0 \\
u_{x x}(0, t)=u_{x x}(32 \pi, t)=-\frac{1}{16^{2}} & , t \geq 0
\end{array}\right\} \\
& \text { مثال (1):الفترة هي [0,32]] والثرط الابتدائي هو[ 10]: } \\
& u(x, 0)=\cos \left(\frac{x}{16}\right)\left(1+\sin \left(\frac{x}{16}\right)\right) \\
& u(x, 0)=c+\frac{15}{19} \sqrt{\frac{11}{19}}\left(-9 \tanh \left(k\left(x-x_{0}\right)\right)\right. \\
& \left.+11 \tanh ^{3}\left(k\left(x-x_{0}\right)\right)\right) \\
& k=\frac{1}{2} \sqrt{\frac{11}{19}}, c=1.2, x_{0}=-12 \text { إذ إن }
\end{aligned}
$$

3- الحل العددي لمعادلة Kuramoto-Sivashinsky:

تعد طريقة الفروقات المنتهية (Finite Differences Method) إحدى أكثر الطرائق الكلاسيكية في

التحليل العددي للمعادلات التفاضليةوتعد إحدى أكثر التطبيقات أهمية, وتُشكل الفروقات المنتهية قاعدة التحليل العددي كما طبقت في طرائق عددية أخرى مثل التفاضل العددي والتكامل العددي. [13,14]

:K.S. لمعادئة (Fully Implicit scheme) اشتقاق صيغة الطريقة الضمنية الكاملة

تسمى الطريقة بالضمنية إذ إن الحل في كل مستوي نتقدم فيه يحتاج إلى حل منظومة معادلات خطية ذات

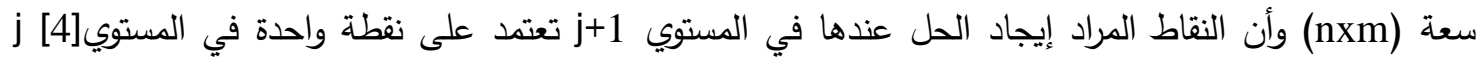
وعلى النحو الآتي: لإيجاد

$$
\begin{aligned}
\frac{u_{i}^{j+1}-u_{i}^{j}}{k}= & -u_{i}^{j}\left[\frac{-3 u_{i}^{j+1}+4 u_{i+1}^{j+1}-u_{i+2}^{j+1}}{2 h}\right]-\left[\frac{2 u_{i}^{j+1}-5 u_{i+1}^{j+1}+4 u_{i+2}^{j+1}-u_{i+3}^{j+1}}{h^{2}}\right] \\
& -\left[\frac{3 u_{i}^{j+1}-14 u_{i+1}^{j+1}+26 u_{i+2}^{j+}-24 u_{i+3}^{j+1}+11 u_{i+4}^{j+1}-2 u_{i+5}^{j+1}}{h^{4}}\right]
\end{aligned}
$$

بضرب المعادلة با ونقل عناصر المستوي (1+1) إلى الطرف الأيمن وعناصر المستوي (j) إلى الطرف الأيسر بفرض أن

$$
\begin{aligned}
u_{i}^{j}=\left(1-\frac{3 h r}{2}\right. & \left.u_{i}^{j}+2 r+\frac{3 r}{h^{2}}\right) u_{i}^{j+1}+\left(-2 r h u_{i}^{j}-5 r-\frac{14 r}{h^{2}}\right) u_{i+1}^{j+1} \\
+ & \left(-\frac{r h}{2} u_{i}^{j}+4 r+\frac{26 r}{h^{2}}\right) u_{i+2}^{j+1}+\left(-r-\frac{24 r}{h^{2}}\right) u_{i+3}^{j+1}+\frac{11 r}{h^{2}} u_{i+4}^{j+1} \\
& -\frac{2 r}{h^{2}} u_{i+5}^{j+1}
\end{aligned}
$$

أما بقية الحلول 


$$
\begin{aligned}
\frac{u_{i}^{j+1}-u_{i}^{j}}{k}=- & u_{i}^{j}\left[\frac{u_{i+1}^{j+1}-u_{i-1}^{j+1}}{2 h}\right]-\left[\frac{u_{i+1}^{j+1}-2 u_{i}^{j+1}+u_{i-1}^{j+1}}{h^{2}}\right] \\
& -\left[\frac{u_{i+2}^{j+1}-4 u_{i+1}^{j+1}+6 u_{i}^{j+1}-4 u_{i-1}^{j+1}+u_{i+2}^{j+1}}{h^{4}}\right]
\end{aligned}
$$

بعد التبسيط تصبح المعادلة أعلاه بالثكل الآتي:

$$
\begin{aligned}
u_{i}^{j}=(1-2 r & \left.+\frac{6 r}{h^{2}}\right) u_{i}^{j+1}+\left(\frac{r h}{2} u_{i}^{j}+r-\frac{4 r}{h^{2}}\right) u_{i+1}^{j+1}+\left(-\frac{r h}{2} u_{i}^{j}+r-\frac{4 r}{h^{2}}\right) u_{i-1}^{j+1} \\
& +\frac{r}{h^{2}} u_{i+2}^{j+1}+\frac{r}{h^{2}} u_{i-2}^{j+1}
\end{aligned}
$$

أما لإيجاد $u_{\mathrm{n}-1}^{\mathrm{j}+1}$ نستخدم تقريبات الفروق التراجعية كما مبين في أدناه:

$$
\begin{aligned}
\frac{u_{i}^{j+1}-u_{i}^{j}}{k}= & -u_{i}^{j}\left[\frac{3 u_{i}^{j+1}-4 u_{i-1}^{j+1}+u_{i-2}^{j+1}}{2 h}\right]-\left[\frac{2 u_{i}^{j+1}-5 u_{i-1}^{j+1}+4 u_{i-2}^{j+1}-u_{i-3}^{j+1}}{h^{2}}\right] \\
& -\left[\frac{3 u_{i}^{j+1}-14 u_{i-1}^{j+1}+26 u_{i-2}^{j+1}-24 u_{i-3}^{j+1}+11 u_{i-4}^{j+1}-2 u_{i-5}^{j+1}}{h^{4}}\right]
\end{aligned}
$$

وعلية نحصل على المعادلة

$$
\begin{aligned}
u_{i}^{j}=\left(1+\frac{3 h r}{2}\right. & \left.u_{i}^{j}+2 r+\frac{3 r}{h^{2}}\right) u_{i}^{j+1}+\left(-2 r h u_{i}^{j}-5 r-\frac{14 r}{h^{2}}\right) u_{i-1}^{j+1} \\
& +\left(\frac{r h}{2} u_{i}^{j}+4 r+\frac{26 r}{h^{2}}\right) u_{i-2}^{j+1}+\left(-r-\frac{24 r}{h^{2}}\right) u_{i-3}^{j+1}+\frac{11 r}{h^{2}} u_{i-4}^{j+1} \\
& -\frac{2 r}{h^{2}} u_{i-5}^{j+1}
\end{aligned}
$$

\section{Boundary conditions الثروط الحدودية}

ذكرنا الأنموذج الرياضي لمعادلة K.S والثروط الحدودية للمعادلة والمتمثلة بالمعادلة b ولتقربب الثروط الحدودية باستخدام الطريقة الصريحة نحتاج إلى تعريف النقاط

$x_{-2}=x_{0}-2 h$

$x_{-1}=x_{0}-h$

$x_{n+1}=x_{n}+h$

$x_{n+2}=x_{n}+2 h$

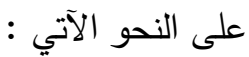

إذ إن هذه النقاط تقع خارج الثبكة وكما في الثكل (1)

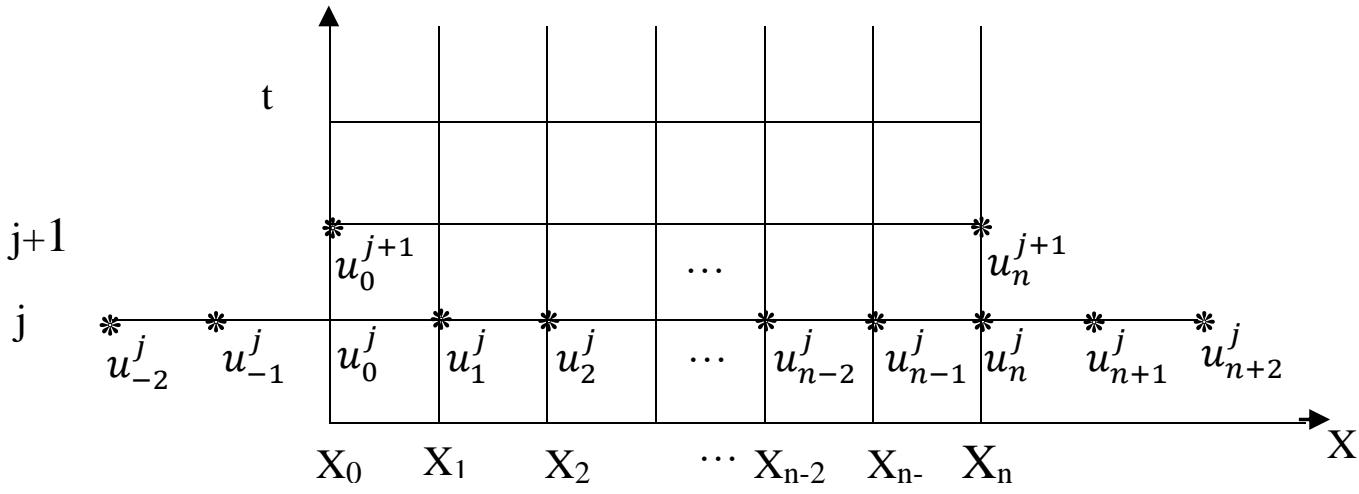




\section{الثكل (1)يوضح النقاط التي تقع خارج المشبك في المستوي ز}

الآن باستخدام الفروقات المركزية للشرط الحدودي الثاني من الرتبة الثانية

$$
\begin{aligned}
& \frac{\partial^{2} u(a, t)}{\partial x^{2}}=-\frac{1}{16^{2}} \\
& \frac{\partial^{2} u(b, t)}{\partial x^{2}}=-\frac{1}{16^{2}}
\end{aligned}
$$

$$
\text { وباستخدام تقريبات الفروقات المركزية للمشتقة الثانية عندما } 0
$$

$\frac{u_{1}^{j}-2 u_{0}^{j}+u_{-1}^{j}}{h^{2}}=-\frac{1}{16^{2}}$
$u_{-1}^{j}=-u_{1}^{j}+2 u_{0}^{j}-\frac{h^{2}}{16^{2}}$

$$
i=n \text { وعندما }
$$

$$
u_{n+1}^{j}=-u_{n-1}^{j}+2 u_{n}^{j}-\frac{h^{2}}{16^{2}} \ldots
$$

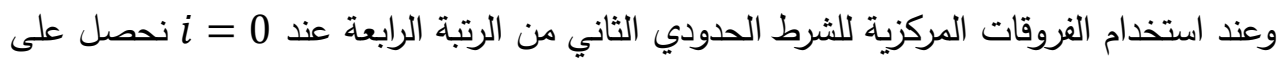

$$
\begin{aligned}
& \frac{-u_{2}^{j}+16 u_{1}^{j}-30 u_{0}^{j}+16 u_{-1}^{j}-u_{-2}^{j}}{12 h^{2}}=-\frac{1}{16^{2}} \\
& u_{-2}^{j}=-u_{2}^{j}+16 u_{1}^{j}-30 u_{0}^{j}+16 u_{-1}^{j}+\frac{12 h^{2}}{16^{2}} \ldots
\end{aligned}
$$

$$
\begin{aligned}
& \quad i=n \text { gعندما } u_{n+2}^{j}=-u_{n-2}^{j}+16 u_{n+1}^{j}-30 u_{n}^{j}+16 u_{n-1}^{j}+\frac{12 h^{2}}{16^{2}} \ldots \text { (11) }
\end{aligned}
$$

نعوض المعادلة (10) في المعادلة (6) عندما 0 = $i=0$ نحصل على المعادلة (12) بعد التبسيط

$$
\begin{aligned}
u_{0}^{j+1}=(1+ & \left.2 r+\frac{24 r}{h^{2}}\right) u_{0}^{j}+\left(-\frac{r h}{2} u_{0}^{j}-r-\frac{12 r}{h^{2}}\right) u_{1}^{j}+\left(\frac{r h}{2} u_{0}^{j}-r-\frac{12 r}{h^{2}}\right) u_{-1}^{j} \\
& -\frac{12 r}{16^{2}} \ldots \text { (12) }
\end{aligned}
$$

نلاحظ أن هناك نقطة أخرى تقع خارج المشبك نعوض المعادلة (8)في المعادلة (12) لنحصل على :

$$
u_{0}^{j+1}=\left(1+r h u_{0}^{j}-\frac{r h_{*}^{3}}{2\left(16^{2}\right)}\right) u_{0}^{j}-\left(r h u_{0}^{j}\right) u_{1}^{j}+\frac{r h^{2}}{16^{2}} \ldots
$$

$$
\text { نعوض المعادلة (11) في المعادلة (6) عندما }
$$$$
u_{n}^{j+1}=\left(1+2 r+\frac{24 r}{h^{2}}\right) u_{n}^{j}+\left(-\frac{r h}{2} u_{n}^{j}-r-\frac{12 r}{h^{2}}\right) u_{n+1}^{j}+\left(\frac{r h}{2} u_{n}^{j}-r-\frac{12 r}{h^{2}}\right) u_{n-1}^{j}
$$$$
-\frac{12 r}{16^{2}}
$$

نلاحظ أيضاً وجود نقطة واقعة خارج حدود المشبك, نعوض المعادلة (9) في المعادلة (14) لنحصل على :

$$
u_{n}^{j+1}=\left(1-r h u_{n}^{j}+\frac{r h^{3}}{2\left(16^{2}\right)}\right) u_{n}^{j}+\left(r h u_{n}^{j}\right) u_{n-1}^{j}+\frac{r h^{2}}{16^{2}} \text {. }
$$

وبعد إيجاد الحل عند الثروط الحدودية نحصل على النظام الخطي الخماسي الأقطار التالي: 


$$
\begin{aligned}
& {\left[\begin{array}{rrrrrrrrr}
A 1 & B 1 & 0 & 0 & \ldots & 0 & 0 & 0
\end{array}\right.} \\
& \begin{array}{llllllllllllll}
0 & A 2 & B 2 & C 1 & D & E & F & F 1 & 0
\end{array} \text {.. } \\
& \begin{array}{llllllll} 
& T H A B & C & 0 & \ldots 0
\end{array} \\
& \begin{array}{llllll}
0 & T H A B C & 0 & . . & 0 & 0
\end{array} \\
& \begin{array}{llllllll}
0 & 0 & T H A & B C & 0 & 0 \ldots 0
\end{array} \\
& \begin{array}{ccc}
0 \ldots 0 & & 0 . .0 \\
0 & . . & 0
\end{array} \\
& =\left[\begin{array}{c}
\left(1-\frac{r h^{3}}{2\left(16^{2}\right)}\right) u_{n}^{j}+\frac{r h^{2}}{16^{2}} \\
u_{1}^{j} \\
\cdot \\
\cdot \\
\cdot \\
\cdot \\
\cdot \\
\cdot \\
u_{n-1}^{j} \\
r h^{3} \\
\left(1+\frac{\left.r 6^{2}\right)}{2(1+2}\right) u_{n}^{j}+\frac{r h^{2}}{16^{2}}
\end{array}\right]
\end{aligned}
$$

$\left.\begin{array}{lll}0 & \ldots & 0\end{array}\right]$

$0 \ldots$

$\cdot\left[\begin{array}{c}u_{0}^{j+1} \\ u_{1}^{j+1} \\ u_{2}^{j+1} \\ \cdot \\ \cdot \\ \cdot \\ u_{n-2}^{j+1} \\ u_{n-1}^{j+1} \\ u_{n}^{j+1}\end{array}\right]$

$\left.\begin{array}{rrrr}T 1 & H 1 & A 3 & 0 \\ 0 & H 2 & A 4\end{array}\right]\left[\begin{array}{l}u_{n-1}^{j+1} \\ u_{n}^{j+1}\end{array}\right.$

$$
\begin{aligned}
& {\left[\begin{array}{llllllll}
A 1 & B 1 & 0 & 0 & \ldots & 0 & 0 & 0
\end{array}\right.} \\
& \begin{array}{lllllllll} 
& A & B & B 2 & C 1 & D 1 & E 1 & F 1 & 0
\end{array} \\
& T H A B C \quad 0 \\
& \text {... } 0 \\
& 0 \ldots 0 \\
& 0 \ldots 0 \\
& =\left[\begin{array}{c}
\left(1-\frac{r h^{3}}{2\left(16^{2}\right)}\right) u_{n}^{j}+\frac{r h^{2}}{16^{2}} \\
u_{1}^{j} \\
\cdot \\
\cdot \\
\cdot \\
\cdot \\
\cdot \\
u_{n-1}^{j} \\
\left(1+\frac{r h^{3}}{2\left(16^{2}\right)}\right) u_{n}^{j}+\frac{r h^{2}}{16^{2}}
\end{array}\right] \\
& \forall j=1,2,3, \ldots, m
\end{aligned}
$$


$\mathrm{A}=\left(1-2 r+\frac{6 r}{h^{2}}\right), \mathrm{A} 1=\left(1-r h u_{n}^{j}\right), \mathrm{A} 2=\left(1-\frac{3 h r}{2} u_{i}^{j}+2 r+\frac{3 r}{h^{2}}\right)$,

$$
\begin{aligned}
& \mathrm{A} 3=\left(1+\frac{3 h r}{2} u_{i}^{j}+2 r+\frac{3 r}{h^{2}}\right), \mathrm{A} 4=\left(1+r h u_{n}^{j}\right), \mathrm{B}=\left(\frac{r h}{2} u_{i}^{j}+r-\frac{4 r}{h^{2}}\right), \mathrm{B} 1=\left(r h u_{n}^{j}\right), \\
& \mathrm{B} 2=\left(-2 r h u_{i}^{j}-5 r-\frac{14 r}{h^{2}}\right), \mathrm{C}=\frac{r}{h^{2}}
\end{aligned}
$$

وبترتيبالمعاد لاتلنحصلعلنظامسداسيالاقطار

$\mathrm{C} 1=\left(-\frac{r h}{2} u_{i}^{j}+4 r+\frac{26 r}{h^{2}}\right), \mathrm{D} 1=\left(-r-\frac{24 r}{h^{2}}\right), \mathrm{D} 2=\left(-r-\frac{24 r}{h^{2}}\right), \mathrm{E} 1=\frac{11 r}{h^{2}}, \mathrm{E} 2=\frac{11 r}{h^{2}}$,

$\mathrm{F} 1=-\frac{2 r}{h^{2}}, \mathrm{~F} 2=-\frac{2 r}{h^{2}}, \mathrm{H}=\left(-\frac{r h}{2} u_{i}^{j}+r-\frac{4 r}{h^{2}}\right), \mathrm{H} 1=\left(-2 r h u_{i}^{j}-5 r-\frac{14 r}{h^{2}}\right), \mathrm{H} 2=\left(-r h u_{n}^{j}\right)$,

$\mathrm{T}=\frac{r}{h^{2}}, \mathrm{~T} 1=\left(\frac{r h}{2} u_{i}^{j}+4 r+\frac{26 r}{h^{2}}\right)$

النظام الخطي أعلاه يمكن إيجاد الحل له باستخدام الطرائق المباشرة (Direct Methods) أو الطرائق

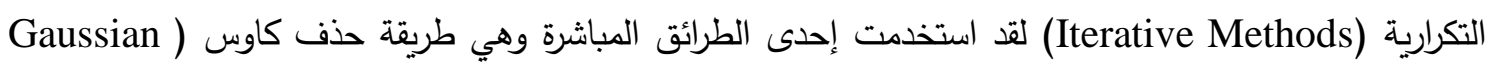

.(Elimination Method

Exponential Finite DifferenceScheme 2-3 اشتقاق صيغة طريقة الفروقات المنتهية الأسية

:K.S. لمعادلة

قدم هذه الطريقة لأول مرة العالم Bhattacharya في عام 1985 للحالة غير المستقرة في البعد الواحد

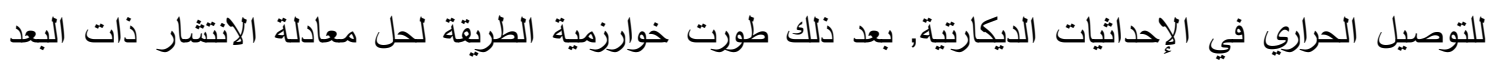
الواحد في الإحداثيات الأسطوانية وطبقت على مسائل ذات بعدين وثلاثة أبعاد. وتستعمل الطريقة لحل المعادلات لاتلات

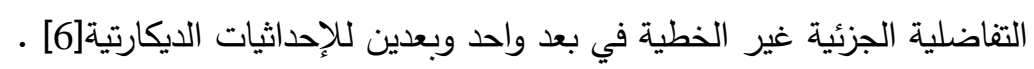

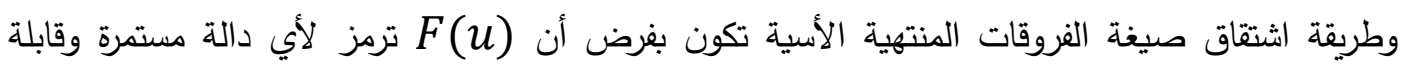

$$
\text { للاشتقاق [2], وبضرب المعادلة (1) بمشتقة F ينتج : }
$$

$$
\frac{\partial F}{\partial t}=F^{\prime}(u)\left(-u \frac{\partial u}{\partial x}-\frac{\partial^{2} u}{\partial x^{2}}-\frac{\partial^{4} u}{\partial x^{2}}\right)
$$

$$
\text { باستخدام الفروقات التقدمية الاعتيادية وتعويضها عن } \frac{\partial F}{\partial t} \text { نحصل على : }
$$

$$
\frac{F\left(u_{i}^{j+1}\right)-F\left(u_{i}^{j}\right)}{k}=F^{\prime}\left(u_{i}^{j}\right)\left[-u_{i}^{j}\left(\frac{\partial u}{\partial x}\right)_{i}^{j}-\left(\frac{\partial^{2} u}{\partial x^{2}}\right)_{i}^{j}-\left(\frac{\partial^{4} u}{\partial x^{4}}\right)_{i}^{j}\right]
$$

نفرض أن $F(u)=\ln u$ نحصل على صيغة الفروقات الأسية وعلى النحو الآتي:

$$
u_{i}^{j+1}=u_{i}^{j} \exp \left[\frac{k}{u_{i}^{j}}\left(-u_{i}^{j}\left(\frac{\partial u}{\partial x}\right)_{i}^{j}-\left(\frac{\partial^{2} u}{\partial x^{2}}\right)_{i}^{j}-\left(\frac{\partial^{4} u}{\partial x^{4}}\right)_{i}^{j}\right)\right]
$$

لإيجاد الحل بهذه الطريقة نتبع الأسلوب المقترح وهو أن يحسب الحل

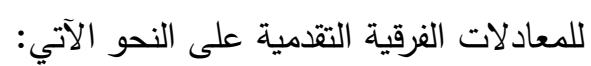

$$
\left(\frac{\partial u}{\partial x}\right)_{i}^{j}=\frac{-3 u_{i}^{j}+4 u_{i+1}^{j}-u_{i+2}^{j}}{2 h}
$$




$$
\begin{aligned}
& \left(\frac{\partial^{2} u}{\partial x^{2}}\right)_{i}^{j}=\frac{2 u_{i}^{j}-5 u_{i+1}^{j}+4 u_{i+2}^{j}-u_{i+3}^{j}}{h^{2}} \\
& \left(\frac{\partial^{4} u}{\partial x^{4}}\right)_{i}^{j}=\frac{3 u_{i}^{j}-14 u_{i+1}^{j}+26 u_{i+2}^{j}-24 u_{i+3}^{j}+11 u_{i+4}^{j}-2 u_{i+5}^{j}}{h^{4}} \\
& \text { وتعويضها في المعادلة(19) نحصل على } \\
& u_{i}^{j+1} \\
& =u_{i}^{j} \exp \left[\frac { k } { u _ { i } ^ { j } } \left(-u_{i}^{j}\left(\frac{-3 u_{i}^{j}+4 u_{i+1}^{j}-u_{i+2}^{j}}{2 h}\right)-\left(\frac{2 u_{i}^{j}-5 u_{i+1}^{j}+4 u_{i+2}^{j}-u_{i+3}^{j}}{h^{2}}\right)\right.\right. \\
& \left.\left.-\left(\frac{3 u_{i}^{j}-14 u_{i+1}^{j}+26 u_{i+2}^{j}-24 u_{i+3}^{j}+11 u_{i+4}^{j}-2 u_{i+5}^{j}}{h^{4}}\right)\right)\right]
\end{aligned}
$$

ولحساب الحلول التقريبية $u\left(x_{i}, t_{j}\right.$ إذ إن i=2,3,..,n-2 نعوض المعادلات الفرقية المركزية في المعادلة

$$
\begin{aligned}
u_{i}^{j+1}=u_{i}^{j} \exp & {\left[\frac { k } { u _ { i } ^ { j } } \left(-u_{i}^{j}\left(\frac{u_{i+1}^{j}-u_{i-1}^{j}}{2 h}\right)-\left(\frac{u_{i+1}^{j}-2 u_{i}^{j}+u_{i-1}^{j}}{h^{2}}\right)\right.\right.} \\
& \left.\left.-\left(\frac{u_{i+2}^{j}-4 u_{i+1}^{j}+6 u_{i}^{j}-4 u_{i-1}^{j}+u_{i-2}^{j}}{h^{4}}\right)\right)\right]
\end{aligned}
$$

أما عند الـ i=n-1 نعوض المعادلات الفرقية الخلفية في المعادلة(19) لنحصل على

$u_{i}^{j+1}$

$$
\begin{aligned}
& =u_{i}^{j} \exp \left[\frac { k } { u _ { i } ^ { j } } \left(-u_{i}^{j}\left(\frac{3 u_{i}^{j}-4 u_{i-1}^{j}+u_{i-2}^{j}}{2 h}\right)-\left(\frac{2 u_{i}^{j}-5 u_{i-1}^{j}+4 u_{i-2}^{j}-u_{i-3}^{j}}{h^{2}}\right)\right.\right. \\
& \left.\left.-\left(\frac{3 u_{i}^{j}-14 u_{i-1}^{j}+26 u_{i-2}^{j}-24 u_{i-3}^{j}+11 u_{i-4}^{j}-2 u_{i-5}^{j}}{h^{4}}\right)\right)\right]
\end{aligned}
$$

\section{Boundary Conditions الثروط الحدودية}

كما موضح في الطريقة الضمنية عند تقريب الثروط الحدودية للمعادلة سيتم الحصول على المعادلات

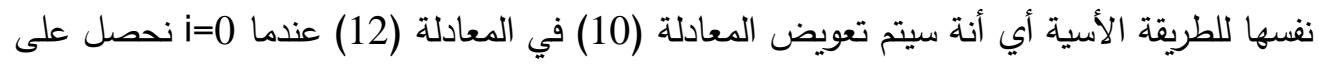

$$
\begin{aligned}
u_{0}^{j+1}=u_{0}^{j} \exp & {\left[\frac { k } { u _ { 0 } ^ { j } } \left(-u_{0}^{j}\left[\frac{u_{1}^{j}-u_{-1}^{j}}{2 h}\right]-\left[\frac{u_{1}^{j}-2 u_{0}^{j}+u_{-1}^{j}}{h^{2}}\right]-\left[\frac{12 u_{1}^{j}-24 u_{0}^{j}+12 u_{-1}^{j}}{h^{4}}\right]\right.\right.} \\
& \left.\left.-\frac{12}{16^{2} h^{2}}\right)\right] \ldots \text { (23) }
\end{aligned}
$$

ونعوض المعادلة (8) في المعادلة (23) لنحصل على

$$
u_{0}^{j+1}=u_{0}^{j} \exp \left[\frac{k}{u_{0}^{j}}\left(\frac{u_{0}^{j}}{2 h}\left(2 u_{1}^{j}+2 u_{0}^{j}-\frac{h^{2}}{16^{2}}\right)-\frac{1}{16^{2}}\right)\right] \ldots
$$




$$
\begin{aligned}
& \text { أما عند i=n نعوض المعادلة (11) في المعادلة (12) نحصل على } \\
& u_{n}^{j+1}=u_{n}^{j} \exp \left[\frac { k } { u _ { n } ^ { j } } \left(-u_{n}^{j}\left[\frac{u_{n+1}^{j}-u_{n-1}^{j}}{2 h}\right]-\left[\frac{u_{n+1}^{j}-2 u_{n}^{j}+u_{n-1}^{j}}{h^{2}}\right]\right.\right. \\
& \left.\left.-\left[\frac{12 u_{n+1}^{j}-24 u_{n}^{j}+12 u_{n-1}^{j}}{h^{4}}\right]-\frac{12}{16^{2} h^{2}}\right)\right] \\
& \text { ونعوض المعادلة (9) في المعادلة (25) لنحصل على } \\
& u_{n}^{j+1}=u_{n}^{j} \exp \left[\frac{k}{u_{n}^{j}}\left(\frac{u_{n}^{j}}{2 h}\left(2 u_{n}^{j}-2 u_{n-1}^{j}-\frac{h^{2}}{16^{2}}\right)-\frac{1}{16^{2}}\right)\right]
\end{aligned}
$$

4- الاستقرارية العددية لمعادلة Kuramoto-Sivashinsky:

تُعد نظرية الاستقرارية العددية للمعادلات التفاضلية الجزئية مع أكثر الشروط الحدودية عمومية في أغلب البعاديه الأحيان صعبة جداً, كالازدواج بين تقطيع الشروط الحدودية وتقطيع المعادلات التفاضلية الجزئية أي يمكن أن

تكون غير ملحوظة [9].

\section{4-1 تحليل الاستقرارية العددية لطريقة الفروقات المنتهية الضمنية الكاملة:}

باستخدام طريقة Fourier(Von-Neumann) نوم بدراسة الاستقرارية العددية لطريقة الفروقات المنتهية

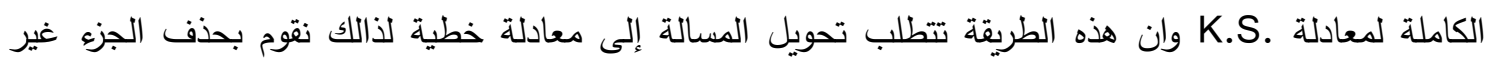

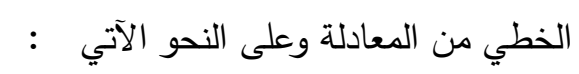

$$
\begin{aligned}
& \frac{u_{i}^{j+1}-u_{i}^{j}}{k} \\
& =-\left[\frac{2 u_{i}^{j+1}-5 u_{i+1}^{j+1}+4 u_{i+2}^{j+1}-u_{i+3}^{j+1}}{h^{2}}\right] \\
& -\left[\frac{3 u_{i}^{j+1}-14 u_{i+1}^{j+1}+26 u_{i+2}^{j+}-24 u_{i+3}^{j+1}+11 u_{i+4}^{j+1}-2 u_{i+5}^{j+1}}{h^{4}}\right]
\end{aligned}
$$

نعوض عن

$$
\begin{aligned}
& \text { طرفي المعادلة بـ } \\
& \frac{\varphi(t+k)}{\varphi(t)}=\frac{1}{\mathrm{a}}
\end{aligned}
$$$$
a=1+r\left[2-5 e^{m \beta h}+4 e^{2 m \beta h}-e^{3 m \beta h}\right]
$$$$
+\frac{r}{h^{2}}\left[3-14 e^{m \beta h}+26 e^{2 m \beta h}-24 e^{3 m \beta h}+11 e^{4 m \beta h}-2 e^{5 m \beta h}\right]
$$$$
\text { باستخدام مفكوك ذي الحدين للمقام وبالتبسيط نحصل على }
$$$$
\frac{\varphi(t+k)}{\varphi(t)}=\frac{1}{\mathrm{a} 1}
$$$$
a 1=1+r\left[\left(1-e^{m \beta h}\right)^{3}+\left(1-e^{m \beta h}\right)^{2}\right]+\frac{r}{h^{2}}\left[2\left(1-e^{m \beta h}\right)^{5}+\left(1-e^{m \beta h}\right)^{4}\right]
$$ 


$$
\begin{aligned}
& \Rightarrow \frac{\varphi(t+k)}{\varphi(t)}=\frac{1}{\mathrm{a} 2} \\
& a 2=1+r\left(1-e^{m \beta h}\right)^{3}\left[1+\frac{2}{h^{2}}\left(1-e^{m \beta h}\right)^{2}\right]+r\left(1-e^{m \beta h}\right)^{2}\left[1+\frac{1}{h^{2}}\left(1-e^{m \beta h}\right)^{2}\right] \\
& \text { بتبسيط المعادلة (28) وإعادة كتابتها على النحو الآتي: } \\
& \frac{\varphi(t+k)}{\varphi(t)}=\frac{1}{\mathrm{~A}+m \mathrm{~B}}=\psi \\
& A=1+r\left(8 r \sin ^{6}\left(\frac{\beta h}{2}\right)-6 \sin ^{2}(\beta h) \sin ^{2}\left(\frac{\beta h}{2}\right)+\frac{64}{h^{2}} \sin ^{10}\left(\frac{\beta h}{2}\right)\right. \\
& -\frac{160}{h^{2}} \sin ^{2}(\beta h) \sin ^{6}\left(\frac{\beta h}{2}\right)+\frac{20}{h^{2}} \sin ^{4}(\beta h) \sin ^{2}\left(\frac{\beta h}{2}\right)+4 \sin ^{4}\left(\frac{\beta h}{2}\right) \\
& \left.-\sin ^{2}(\beta h)+\frac{16}{h^{2}} \sin ^{8}\left(\frac{\beta h}{2}\right)-\frac{24}{h^{2}} \sin ^{2}(\beta h) \sin ^{4}\left(\frac{\beta h}{2}\right)+\frac{1}{h^{2}} \sin ^{4}(\beta h)\right) \\
& B=r \sin (\beta h)\left(-12 \sin ^{4}\left(\frac{\beta h}{2}\right)+\sin ^{2}(\beta h)-\frac{160}{h^{2}} \sin ^{8}\left(\frac{\beta h}{2}\right)+\frac{80}{h^{2}} \sin ^{2}(\beta h) \sin ^{4}\left(\frac{\beta h}{2}\right)\right. \\
& -\frac{2}{h^{2}} \sin ^{4}(\beta h)-4 \sin ^{2}\left(\frac{\beta h}{2}\right)-\frac{32}{h^{2}} \sin ^{6}\left(\frac{\beta h}{2}\right) \\
& \left.+\frac{8}{h^{2}} \sin ^{2}(\beta h) \sin ^{2}\left(\frac{\beta h}{2}\right)\right)
\end{aligned}
$$

إن الشرط الضروري والكافي للاستقرارية العددية هو$$
\left|\frac{\varphi(t+k)}{\varphi(t)}\right|=|\psi| \leq 1
$$$$
\text { لبعض قيم (Bh) تكون } 1 \text { = } 18 \sin ^{2}\left(\frac{\beta h}{2}\right) \text { نحصل على }
$$$$
A=1+r\left(5-\frac{83}{h^{2}}\right)
$$$$
B=r \sin (\beta h)\left(-15-\frac{106}{h^{2}}\right)
$$

$$
|\psi|=\frac{1}{\sqrt{\mathrm{A}^{2}+\mathrm{B}^{2}}}=\frac{1}{\sqrt{1+2 r\left(5-\frac{83}{h^{2}}\right)+r^{2}\left(5-\frac{83}{h^{2}}\right)^{2}+r^{2}\left(-15-\frac{106}{h^{2}}\right)^{2}}}
$$

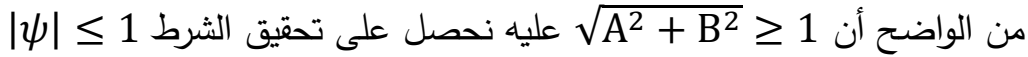

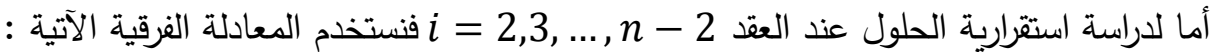

$$
\begin{aligned}
& \frac{u_{i}^{j+1}-u_{i}^{j}}{k}=-\left[\frac{u_{i+1}^{j+1}-2 u_{i}^{j+1}+u_{i-1}^{j+1}}{h^{2}}\right]-\left[\frac{u_{i+2}^{j+1}-4 u_{i+1}^{j+1}+6 u_{i}^{j+1}-4 u_{i-1}^{j+1}+u_{i+2}^{j+1}}{h^{4}}\right] \\
& \text { نعوض عن } \\
& \frac{\varphi(t+k)}{\varphi(t)}=\frac{1}{\mathrm{a}} \\
& \mathrm{a}=1+r\left[e^{m \beta h}-2+e^{-m \beta h}\right]+\frac{r}{h^{2}}\left[e^{2 m \beta h}-4 e^{m \beta h}+6-4 e^{-m \beta h}+e^{-2 m \beta h}\right]
\end{aligned}
$$




$$
\begin{aligned}
& \frac{\varphi(t+k)}{\varphi(t)}=\frac{1}{\mathrm{a} 1}=\frac{1}{\mathrm{~A}}=\psi \\
& \mathrm{a} 1=1+4 r \sin ^{2}\left(\frac{\beta h}{2}\right)-\frac{8 r}{h^{2}} \sin ^{4}\left(\frac{\beta h}{2}\right)+\frac{2 r}{h^{2}} \sin ^{2}(\beta h)-\frac{8 r}{h^{2}} \sin ^{2}\left(\frac{\beta h}{2}\right) \\
& \text { لبعض قيم } \\
& |\psi|=\frac{1}{\sqrt{A^{2}}}=\frac{1}{\sqrt{1+\mathrm{r}\left(-8+\frac{28}{h^{2}}\right)+r^{2}\left(4-\frac{14}{h^{2}}\right)^{2}}}
\end{aligned}
$$

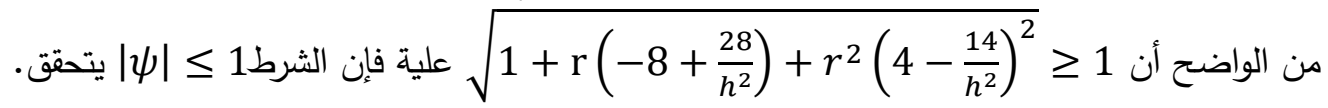

$$
\begin{aligned}
& \text { أما النقطة i=n-n فنستخدم المعادلة الفرقية الآتية : } \\
& \frac{u_{i}^{j+1}-u_{i}^{j}}{k} \\
& =-\left[\frac{2 u_{i}^{j+1}-5 u_{i-1}^{j+1}+4 u_{i-2}^{j+1}-u_{i-3}^{j+1}}{h^{2}}\right] \\
& -\left[\frac{3 u_{i}^{j+1}-14 u_{i-1}^{j+1}+26 u_{i-2}^{j+1}-24 u_{i-3}^{j+1}+11 u_{i-4}^{j+1}-2 u_{i-5}^{j+1}}{h^{4}}\right] \\
& \text { نعوض عن } \\
& \frac{\varphi(t+k)}{\varphi(t)}=\frac{1}{\mathrm{a}} \\
& a=1+r\left[2-5 e^{-m \beta h}+4 e^{-2 m \beta h}-e^{-3 m \beta h}\right] \\
& +\frac{r}{h^{2}}\left[3-14 e^{-m \beta h}+26 e^{-2 m \beta h}-24 e^{-3 m \beta h}+11 e^{-4 m \beta h}\right. \\
& \left.-2 e^{-5 m \beta h}\right] \\
& \text { باستخدام مفكوك ذي الحدين للمقام وبالتبسيط نحصل على } \\
& \frac{\varphi(t+k)}{\varphi(t)}=\frac{1}{\mathrm{a} 1} \\
& a 1=1+r\left[\left(1-e^{-m \beta h}\right)^{3}+\left(1-e^{-m \beta h}\right)^{2}\right]+\frac{r}{h^{2}}\left[2\left(1-e^{-m \beta h}\right)^{5}+\left(1-e^{-m \beta h}\right)^{4}\right] \\
& \Rightarrow \frac{\varphi(t+k)}{\varphi(t)}=\frac{1}{\mathrm{a} 2} \\
& a 2=1+r\left(1-e^{-m \beta h}\right)^{3}\left[1+\frac{2}{h^{2}}\left(1-e^{-m \beta h}\right)^{2}\right] \\
& +r\left(1-e^{-m \beta h}\right)^{2}\left[1+\frac{1}{h^{2}}\left(1-e^{-m \beta h}\right)^{2}\right] \\
& \text { بتبسيط المعادلة (32) وإعادة كتابتها على النحو الآتي: } \\
& \frac{\varphi(t+k)}{\varphi(t)}=\frac{1}{\mathrm{~A}+\mathrm{mB}}=\psi
\end{aligned}
$$

إذ إن ش هو عامل التضخم وإن 


$$
\begin{aligned}
& A=1+r\left(8 r \sin ^{6}\left(\frac{\beta h}{2}\right)-6 \sin ^{2}(\beta h) \sin ^{2}\left(\frac{\beta h}{2}\right)+\frac{64}{h^{2}} \sin ^{10}\left(\frac{\beta h}{2}\right)\right. \\
& -\frac{160}{h^{2}} \sin ^{2}(\beta h) \sin ^{6}\left(\frac{\beta h}{2}\right)+\frac{20}{h^{2}} \sin ^{4}(\beta h) \sin ^{2}\left(\frac{\beta h}{2}\right)+4 \sin ^{4}\left(\frac{\beta h}{2}\right) \\
& \left.-\sin ^{2}(\beta h)+\frac{16}{h^{2}} \sin ^{8}\left(\frac{\beta h}{2}\right)-\frac{24}{h^{2}} \sin ^{2}(\beta h) \sin ^{4}\left(\frac{\beta h}{2}\right)+\frac{1}{h^{2}} \sin ^{4}(\beta h)\right) \\
& B=r \sin (\beta h)\left(12 \sin ^{4}\left(\frac{\beta h}{2}\right)-\sin ^{2}(\beta h)+\frac{160}{h^{2}} \sin ^{8}\left(\frac{\beta h}{2}\right)-\frac{80}{h^{2}} \sin ^{2}(\beta h) \sin ^{4}\left(\frac{\beta h}{2}\right)\right. \\
& +\frac{2}{h^{2}} \sin ^{4}(\beta h)+4 \sin ^{2}\left(\frac{\beta h}{2}\right)+\frac{32}{h^{2}} \sin ^{6}\left(\frac{\beta h}{2}\right) \\
& \left.-\frac{8}{h^{2}} \sin ^{2}(\beta h) \sin ^{2}\left(\frac{\beta h}{2}\right)\right)
\end{aligned}
$$

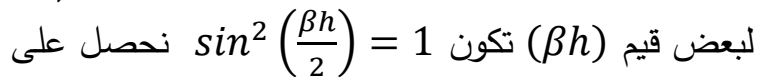

$$
\begin{aligned}
& A=1+r\left(5-\frac{83}{h^{2}}\right) \\
& B=r \sin (\beta h)\left(15+\frac{106}{h^{2}}\right) \\
& |\psi|=\frac{1}{\sqrt{\mathrm{A}^{2}+\mathrm{B}^{2}}}=\frac{1}{\sqrt{1+2 r\left(5-\frac{83}{h^{2}}\right)+r^{2}\left(5-\frac{83}{h^{2}}\right)^{2}+r^{2}\left(15+\frac{106}{h^{2}}\right)^{2}}}
\end{aligned}
$$

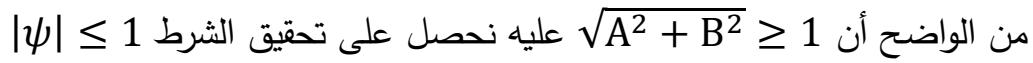

عليه فإن الطريقة الضمنية الكاملة (Fully-Implicit Method) تكون مستقرة بدون شرط الفيل عند جميع نقاط المشبك. (unconditionally stable)

Fourier (Von- ت 4-2 الاسيل الاسترارية العددية لطريقة الفروقات المنتهية الأسية باستخدام طريقة

: Neumann)

لدراسة الاستقرارية العددية لطريقة الفروقات الأسية نقوم بحذف الجزء غير الخطي من المعادلة (19) . المعادلة مع الجزء غير الخطي هي : $u_{i}^{j+1}=u_{i}^{j} \exp \left[\frac{k}{u_{i}^{j}}\left(-\mathrm{u}_{\mathrm{i}}^{\mathrm{j}}\left(\frac{\partial \mathrm{u}}{\partial \mathrm{x}}\right)_{\mathrm{i}}^{\mathrm{j}}-\left(\frac{\partial^{2} \mathrm{u}}{\partial \mathrm{x}^{2}}\right)_{\mathrm{i}}^{\mathrm{j}}-\left(\frac{\partial^{4} \mathrm{u}}{\partial \mathrm{x}^{4}}\right)_{\mathrm{i}}^{\mathrm{j}}\right)\right]$

بحذف الجزء غير الخطي من المعادلة (34) يكون لدينا :

$$
u_{i}^{j+1}=u_{i}^{j} \exp \left[\frac{k}{u_{i}^{j}}\left(-\left(\frac{\partial^{2} \mathrm{u}}{\partial \mathrm{x}^{2}}\right)_{\mathrm{i}}^{\mathrm{j}}-\left(\frac{\partial^{4} \mathrm{u}}{\partial \mathrm{x}^{4}}\right)_{\mathrm{i}}^{\mathrm{j}}\right)\right]
$$

ولدراسة الاستقرارية العددية نبدأ بالعقدة الثانية عندما i=1 نستخدم المعادلة الفرقية الآتية: 


$$
\begin{aligned}
& u_{\mathrm{i}}^{\mathrm{j}+1} \\
& =u_{\mathrm{i}}^{\mathrm{j}} \exp \left[\frac { \mathrm { k } } { u _ { \mathrm { i } } ^ { \mathrm { j } } } \left(-\left(\frac{2 u_{i}^{j}-5 u_{i+1}^{j}+4 u_{i+2}^{j}-u_{i+3}^{j}}{\mathrm{~h}^{2}}\right)\right.\right. \\
& \left.\left.-\left(\frac{3 \mathrm{u}_{\mathrm{i}}^{\mathrm{j}}-14 \mathrm{u}_{\mathrm{i}+1}^{\mathrm{j}}+26 \mathrm{u}_{\mathrm{i}+2}^{\mathrm{j}}-24 \mathrm{u}_{\mathrm{i}+3}^{\mathrm{j}}+11 \mathrm{u}_{\mathrm{i}+4}^{\mathrm{j}}-2 \mathrm{u}_{\mathrm{i}+5}^{\mathrm{j}}}{\mathrm{h}^{4}}\right)\right)\right]
\end{aligned}
$$

نعوض عن

$$
\frac{\varphi(t+k)}{\varphi(t)}=\exp \left\{r\left(1-e^{m \beta h}\right)^{3}\left[-1-\frac{2}{h^{2}}\left(1-e^{m \beta h}\right)^{2}\right]\right.
$$

$$
\left.+r\left(1-e^{m \beta h}\right)^{2}\left[-1-\frac{1}{h^{2}}\left(1-e^{m \beta h}\right)^{2}\right]\right\}
$$

وبتبسيط المعادلة أعلاه وبالأسلوب المتبع نفسه في الطرائق الأخرى نحصل على :

$$
\frac{\varphi(t+k)}{\varphi(t)}=\exp \{\mathrm{A}+\mathrm{mB}\}=\psi
$$

$$
\begin{aligned}
& A=r\left(-5+\frac{83}{h^{2}}\right) \\
& B=r \sin (\beta h)\left(15+\frac{106}{h^{2}}\right) \\
& |\psi|=|\exp \{A+i B\}|=e^{A} \leq 1 \\
& \text { عليه تكون } 1 \text { | }|\psi| \text { فقط إذا كانت } 0 \text { أي } 1 \text { أي } \\
& r\left(-5+\frac{83}{h^{2}}\right) \leq 0 \\
& \text { بما أن } 0 \text { > } \\
& -5+\frac{83}{h^{2}} \leq 0 \Rightarrow \frac{83}{h^{2}} \leq 5 \Rightarrow h>\sqrt{\frac{83}{5}} \\
& u_{\mathrm{i}}^{\mathrm{j}+1}=u_{\mathrm{i}}^{\mathrm{j}} \exp \left[\frac { \mathrm { k } } { u _ { \mathrm { i } } ^ { \mathrm { j } } } \left(-\left(\frac{u_{i+1}^{j}-2 u_{i}^{j}+u_{i-1}^{j}}{\mathrm{~h}^{2}}\right)\right.\right. \\
& \left.\left.-\left(\frac{u_{i+2}^{j}-4 u_{i+1}^{j}+6 u_{i}^{j}-4 u_{i-1}^{j}+u_{i-2}^{j}}{h^{4}}\right)\right)\right] \\
& \frac{\varphi(t+k)}{\varphi(t)}=\exp \left\{-r\left[e^{m \beta h}-2+e^{-m \beta h}\right]\right. \\
& \left.-\frac{r}{h^{2}}\left[e^{2 m \beta h}-4 e^{m \beta h}+6-4 e^{-m \beta h}+e^{-2 m \beta h}\right]\right\}
\end{aligned}
$$




$$
\begin{aligned}
& \frac{\varphi(t+k)}{\varphi(t)}=\exp \left\{4 r \sin ^{2}\left(\frac{\beta h}{2}\right)-\frac{8 r}{h^{2}} \sin ^{4}\left(\frac{\beta h}{2}\right)+\frac{2 r}{h^{2}} \sin ^{2}(\beta h)-\frac{8 r}{h^{2}} \sin ^{2}\left(\frac{\beta h}{2}\right)\right\}=e^{A} \\
& =\psi \\
& A=4 r \sin ^{2}\left(\frac{\beta h}{2}\right)-\frac{8 r}{h^{2}} \sin ^{4}\left(\frac{\beta h}{2}\right)+\frac{2 r}{h^{2}} \sin ^{2}(\beta h)-\frac{8 r}{h^{2}} \sin ^{2}\left(\frac{\beta h}{2}\right) \\
& |\psi|=\left|e^{A}\right| \\
& \text { لكي يتحقق الشرط يجب أن تكون } 0 \leq \text { أي أن } \\
& A=\mathrm{r}\left(4-\frac{14}{h^{2}}\right) \\
& r\left(4-\frac{14}{h^{2}}\right) \leq 0 \\
& \text { بما أن } 0 \text { r } 0 \\
& 4-\frac{14}{h^{2}} \leq 0 \Rightarrow \frac{14}{h^{2}} \leq 4 \\
& \Rightarrow \frac{1}{h^{2}} \leq \frac{4}{14} \Rightarrow h \geq \sqrt{\frac{14}{4}}
\end{aligned}
$$

أما للعقدة i=n-1 فنستخدم المعادلة الفرقية الآتية :

$$
\begin{aligned}
& u_{i}^{j+1} \\
& =u_{i}^{j} \exp \left[\frac { k } { u _ { i } ^ { j } } \left(-\left(\frac{2 u_{i}^{j}-5 u_{i-1}^{j}+4 u_{i-2}^{j}-u_{i-3}^{j}}{h^{2}}\right)\right.\right. \\
& \left.\left.-\left(\frac{3 u_{i}^{j}-14 u_{i-1}^{j}+26 u_{i-2}^{j}-24 u_{i-3}^{j}+11 u_{i-4}^{j}-2 u_{i-5}^{j}}{h^{4}}\right)\right)\right] \\
& \frac{\varphi(t+k)}{\varphi(t)}=\exp \left\{-r\left[2-5 e^{-m \beta h}+4 e^{-2 m \beta h}-e^{-3 m \beta h}\right]\right. \\
& -\frac{r}{h^{2}}\left[3-14 e^{-m \beta h}+26 e^{-2 m \beta h}-24 e^{-3 m \beta h}+11 e^{-4 m \beta h}\right. \\
& \left.\left.-2 e^{-5 m \beta h}\right]\right\} \\
& \text { باستخدام مفكوك ذي الحدين نحصل على } \\
& \frac{\varphi(t+k)}{\varphi(t)}=\exp \left\{r\left(1-e^{-m \beta h}\right)^{3}\left[-1-\frac{2}{h^{2}}\left(1-e^{-m \beta h}\right)^{2}\right]\right. \\
& \left.+r\left(1-e^{-m \beta h}\right)^{2}\left[-1-\frac{1}{h^{2}}\left(1-e^{-m \beta h}\right)^{2}\right]\right\}
\end{aligned}
$$

وبتبسيط المعادلة أعلاه بالأسلوب المتبع نفسه في الطرائق الأخرى نحصل على

$$
\frac{\varphi(t+k)}{\varphi(t)}=\exp \{\mathrm{A}+\mathrm{mB}\}=\psi
$$




$$
\begin{aligned}
& A=r\left(-5+\frac{83}{h^{2}}\right) \\
& B=r \sin (\beta h)\left(-15-\frac{106}{h^{2}}\right) \\
& |\psi|=|\exp \{A+m B\}|=e^{A} \leq 1 \\
& \text { من المعادلة (41) نجد أن } \\
& \text { بما أن } 0 \text { > } 0 \text { دائماً صحيحة عليه } \\
& -5+\frac{83}{h^{2}} \leq 0 \Rightarrow \frac{1}{h^{2}} \leq \frac{5}{83} \Rightarrow h \geq \sqrt{\frac{83}{5}}
\end{aligned}
$$

للخطوة الزمنية KXxponential Finite difference لحل معادلة

تكون غير مشروطة (unconditionally stable) لكل النقاط.

Numerical Results النتائج العددية

سنناقش في هذه الفقرة النتائج العددية لطرائق الفروقات المنتهية المتمثلة بالطرائق الآتية الطريقة الضمنية

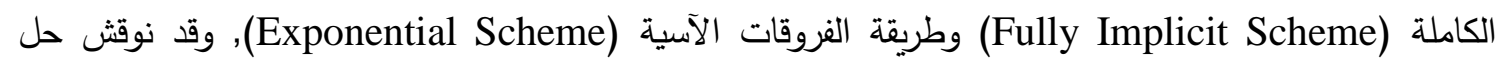

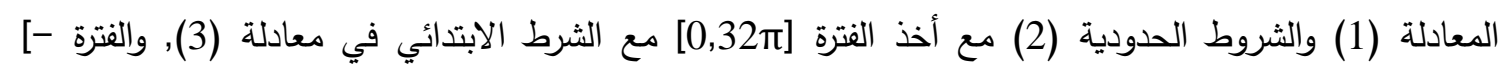

30,30] الثرط الابتدائي في معادلة (4) :

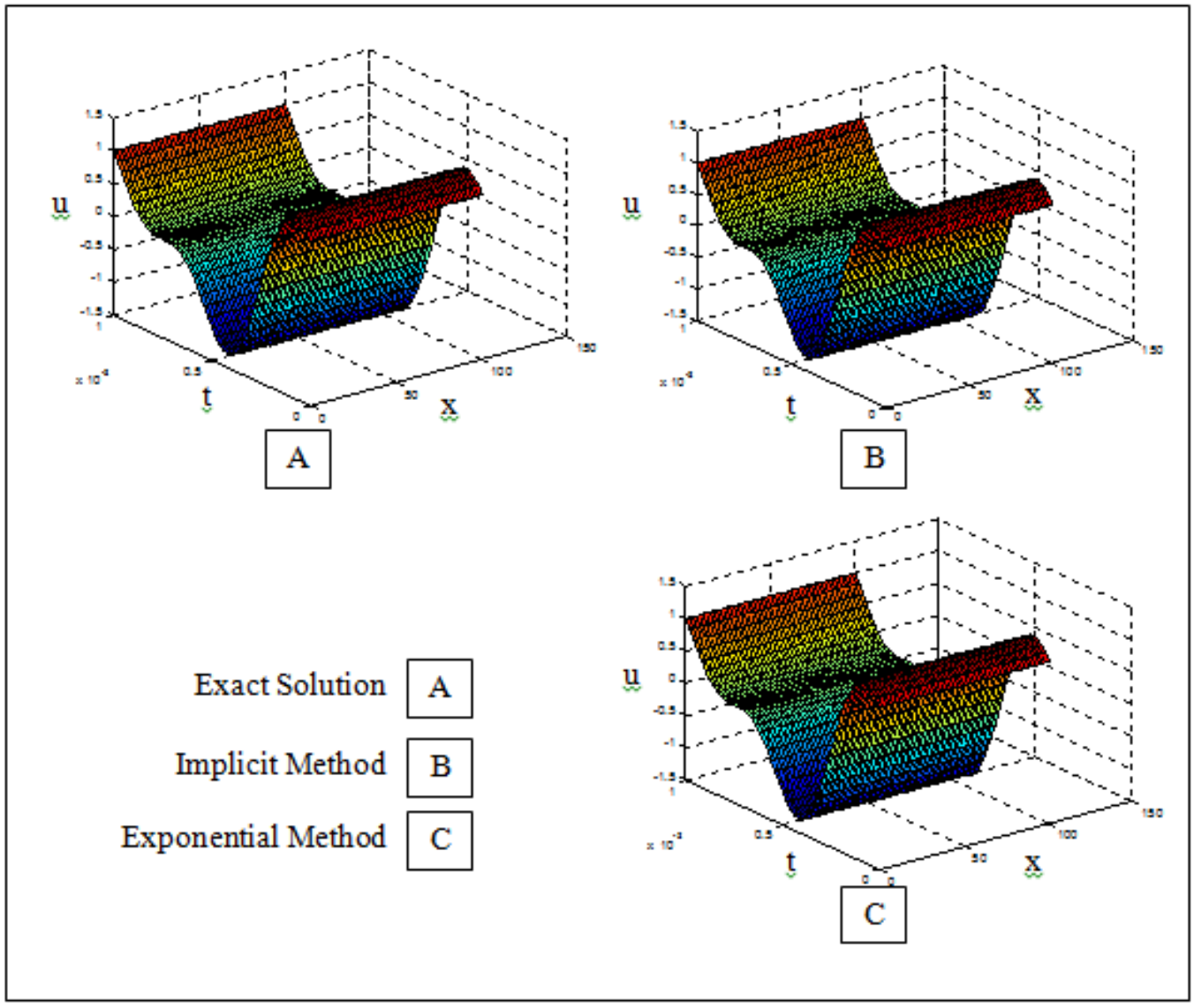

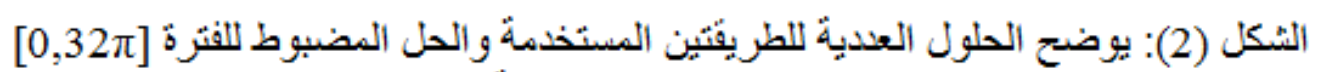

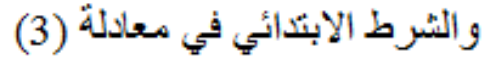




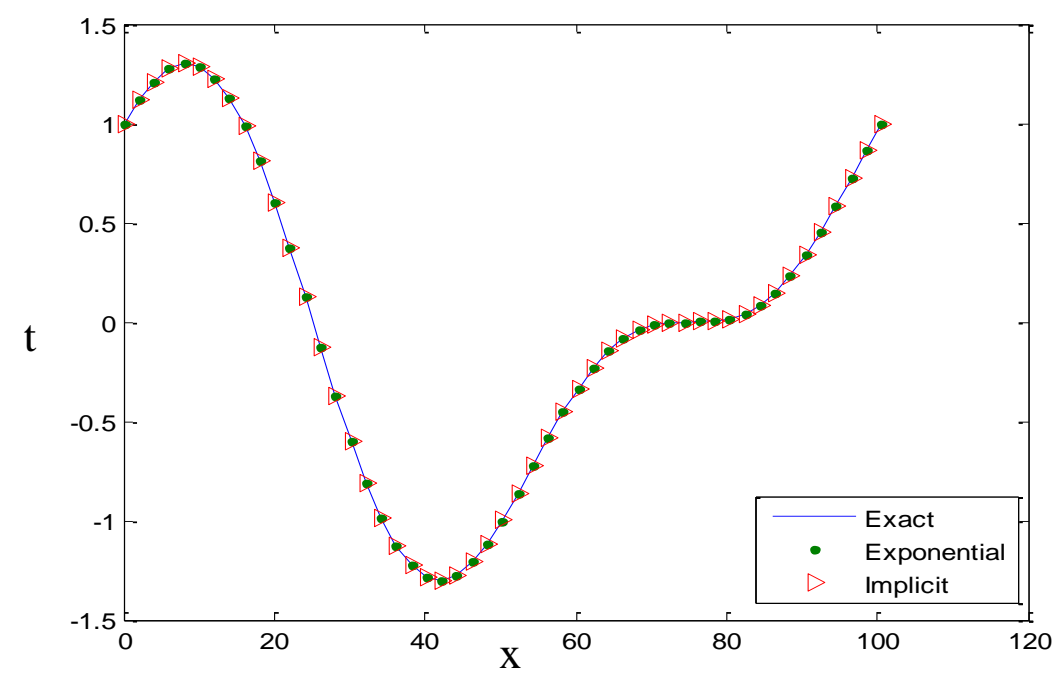

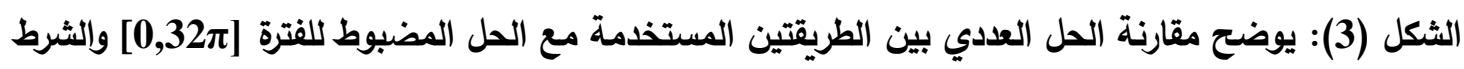

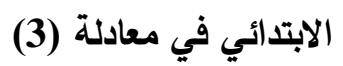

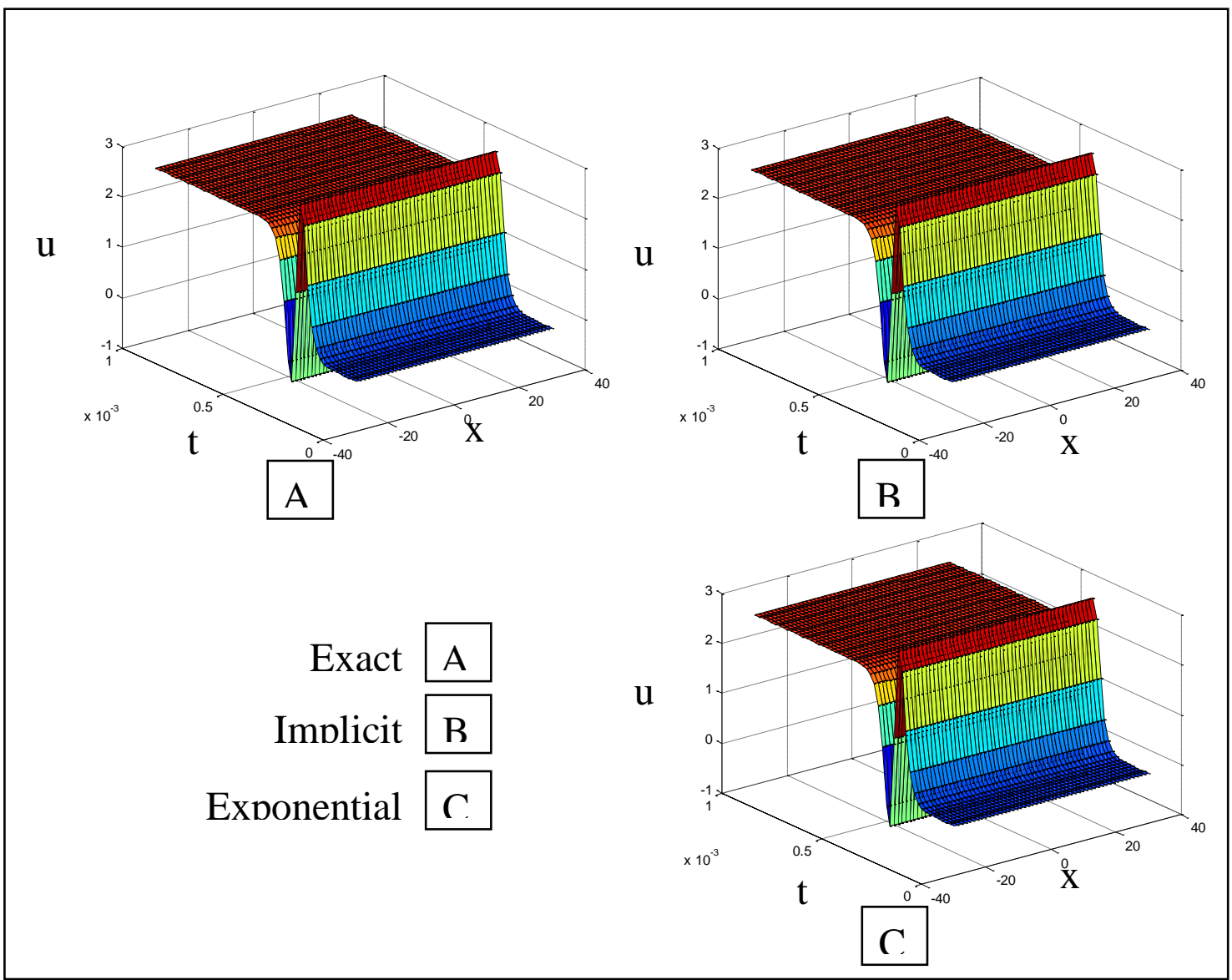

الثكل(4): يوضح الحلول العددية للطريقتين المستخدمة والحل المضبوط للفترة [30,30-] والثرط الابتدائي في معادلة (4) 


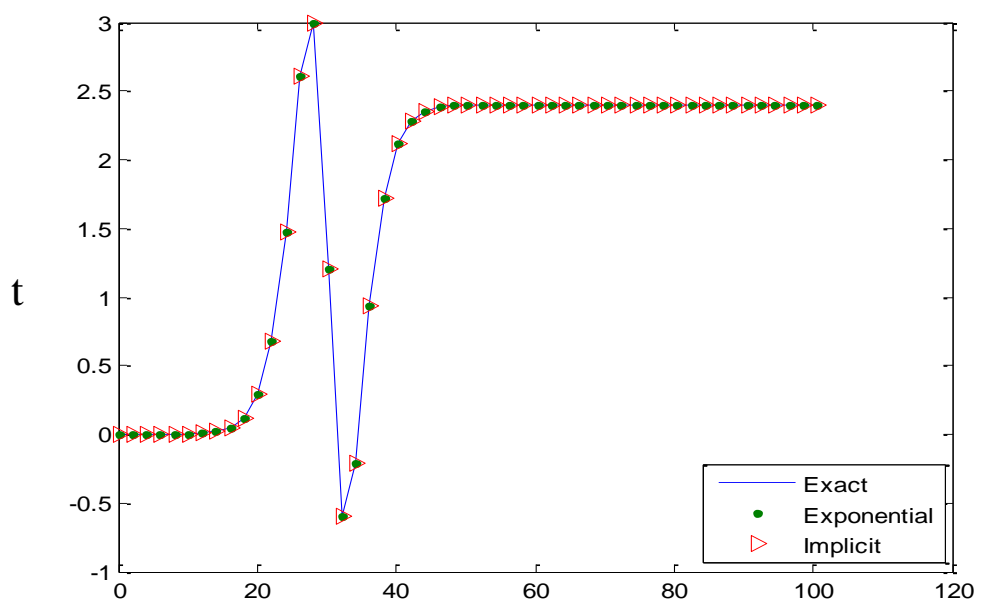

الثكل (5): يوضح مقارنة الحل العددي بين الطريقتين المستخدمة مع الحل المضبوط للفترة[130,30] والثرط الابتدائي في معادلة (4) بن بن

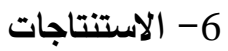

من خلال دراستتا لمعادلة K.S. لاحظنا أنها من المعادلات الحرارية المهمة في التطبيقات الهندسية والفيزيائية, وقد استخدمها العلماء بكثرة في الآونة الأخيرة, وتعد من المعادلات غير الخطية التي تحتاج إلى جهد كبير لغرض إيجاد الحل العددي لها وتحتاج دقة كبيرة في معالجة المشتقة الرابعة, وقد توصلنا إلى أهم الاستتتاجات التي هي: unconditionally ( إن الطريقة الضمنية الكاملة (Fully-Implicit Method) تكون مستقرة بدون شرط (stable أن طريقة الفروقات الأسية Exponential Finite difference للحطوة الزمنية k.S.S. لكون معادلة غير مشروطة (unconditionally stable) لكل النقاط. 


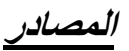

[1] Al-Rawi, Ekhlass S. and Al-Baker, Al-Moutasam A., (2011), "Finite Difference Method to Solve Korteweg-de Vries-Burger Equation", Al-Rafiden J. of Com. Sci. and Math., Vol.8, No.1, PP.65-80.

[2] Bahadir, A. Refik, (2005), "Exponential Finite-Difference Method Applied to Korteweg-de Vries Equation for Small Times", Applied Mathematics and Computation, Vol. 160, PP. 675-682.

[3] Dubljevic S., (2010), "Boundary Model Predictive Control of KuramotoSivashinskyEquation with Input and State Constraints", Computers and Chemical Engineering, Vol.34, PP.1655-1661.

[4] Duffy, Daniel J. (2006), "Finite Difference Methods in Financial Engineering A Partial Differential Equation Approach", England,the Atrium, Southern Gate, Chi-Chester, West Sussex PO19 8SQ, John Wiley \&Sons Ltd.

[5] Hag F. I., (2009), "Numerical Solution of Boundary-Value and InitialBoundary-Value Problems using Spline Fuctions", Topi, University of Pakistan, Master Thesis.

[6] Handschuh F. R. and Keith G. T., (1988), "Applications of an Exponential Finite Difference Technique", NASA, Technical Memorandum 100939, AVSCOM, Technical Memorandum 88-C-004.

[7] Kudryashov N. A., (2004), "Simplest Equation Method to look for Exact Solutions of Nonlinear Differential Equations",Departmentof Applied MathematicsMoscowEngineering and Physics Institute.http://arXiv.org/abs/nlin/0406007v1

[8] Lapidus, Leon and George, F.P., (1982), "Numerical Solution of Parital Differential Equation in Science and Engineering", John Wiley and Sons, Inc.

[9] Leveque J. R., (2007), "Finite Difference Method for Ordinary and Partial Differential Equations" Society for industrial and Applied Mathematics.

[10] Halifax, Nova Scotia, (2009), "High Order Collocation Software forthe Numerical Solution of Fourth Order Parabolic PDEs", Ling Lin, University of Saint Mary's, Master Thesis.

[11] MacKenzie T. and Roberts A. J., (2008), "Holistic Finite Differences Accurately Model the Dynamics of the Kuramoto-Sivashinsky Equation", Dept Maths \& Comp, University of Southern Qld.http://arXiv.org/abs/math/0001079v2.

[12] Sastry S. S., (2010), "Introductory Methods of Numerical Analysis", Fourth Edition, PHI Learning Private Limited, New Delhi.

[13] Steven T. Karris, (2007), "Numerical Analysis Using Matlab and Excel", Third 
Edition, Orchard Publications.

[14] Ulf R. K. and Erlend M. V.,(2010), "Computational Methods in Acovstic", Department of Electronics and Telecommunications-NANU.

[15] Wazwaz, A. M., (209), "Partial Differential Equations and Solitary Waves Theory", Higher Eduction Press, Beijing and Springer-Verlag Berlin Heidelberg. 